Frank Zöllner

\title{
Die Bedeutung von Codex Huygens und Codex Urbinas für die Proportions- und Bewegungsstudien Leonardos da Vinci
}

Erstmals publiziert in:

Zeitschrift für Kunstgeschichte, 52, 1989, S. 334-352.

Hinweis:

Die Seitenzählung der vorliegenden elektronischen Version weicht von der Paginierung der Druckfassung ab.

Zitierfähige URL:

http://archiv.ub.uni-heidelberg.de/artdok/volltexte/2006/155/ 


\section{Frank Zöllner}

\section{Die Bedeutung von Codex Huygens und Codex Urbinas für die Proportions- und Bewegungsstudien Leonardos da Vinci}

$\mathrm{Zu}$ den wichtigsten thematischen Schwerpunkten, unter denen die bildende Kunst des 15. und 16. Jahrhunderts erforscht wird, gehört neben der Perspektive, der antiken Mythologie und der christlichen Ikonographie die Lehre von den Proportionen und Bewegungen des menschlichen Körpers. Ihre grundsätzliche Bedeutung ist - allerdings weitgehend unter Ausschluß der Bewegungslehre bereits 1921 von Erwin Panofsky in einem bis heute maßgeblichen Aufsatz dargelegt und seitdem oft diskutiert worden. ${ }^{1}$ Ebenso haben die umfangreichsten und wichtigsten Proportionsstudien der Renaissance, nämlich diejenigen Leonardo da Vincis ${ }^{2}$ und Albrecht Dürers ${ }^{3}$ inzwischen eine wissenschaftliche Würdigung gefunden. Andererseits wurde gerade die Bewegungstheorie Leonardos weder hinsichtlich ihrer eigenen Chronologie noch im Hinblick auf die allgemeine Entwicklungsgeschichte der Proportions- und Bewegungslehre ausreichend und zusammenhängend analysiert. ${ }^{4}$ Die Gründe hierfür sind der Verlust des größten Teils der Bewegungsstudien sowie das hoffnungslose Durcheinander und die verzwickte Überlieferungsgeschichte der Manuskripte Leonardos. Gegenstand des folgenden Aufsatzes ist daher die chronologische und inhaltliche Rekonstruktion und Zusammenstellung sowohl verlorenen als auch erhaltenen Materials, um mit dessen Hilfe eine allgemeinere Einschätzung und Analyse der Proportions- und Bewegungsstudien Leonardos zu ermöglichen. Dabei dienen zwei im unterschiedlichen Maße als leonardesk geltende Manuskripte als Ausgangspunkt: der Codex Huygens und der Codex Urbinas.

I.

Leonardos literarisches Erbe, seine theoretischen Arbeiten und seine Zeichnungen finden sich auf rund 6500 erhaltenen Manuskriptblättern, die meistens jede Form von inhaltlicher oder chronologischer Ordnung vermissen lassen. Weiteres Material, das einen Umfang von schätzungsweise 2000 bis 5000 Blättern gehabt haben kann, muß heute als verloren gelten. ${ }^{5}$ Aufgrund der hohen Verlustrate von möglicherweise 50\% kommt den Kompilationen, die noch während des 16. Jahrhunderts nach heute verlorenen Originalen entstanden sind, eine besondere Bedeutung zu. Die wichtigsten Kompilationen dieser Art sind der als "Trattato della pittura" oder "Libro della pittura" bekannte Codex Urbinas 1270 der Biblioteca Apostolica Vaticana in Rom und der Codex Huygens (Cod. M.A. 1139) der Pierpont Morgan Library in New York. Beide Codices enthalten unter anderem Material zu den Proportionen und Bewegungen des menschlichen Körpers: der Codex Urbinas auf den ersten 40 Folios seines dritten Abschnitts und der Codex Huygens an drei verschiedenen Stellen: Zum einen finden sich auf den ersten 30 Blättern geometrisch 
schematisierte Bewegungsstudien, um die es im folgenden hauptsächlich gehen wird (Abb. 4,6,7,8). Zum anderen handelt es sich, auf den fols. 39 bis 52, um eine Gruppe von Studien, die (möglicherweise auf ein Programm Leonardos zurückgehend ${ }^{6}$ ) den Proportionen des menschlichen Körpers differenziert nach Alter, Größe und Geschlecht gewidmet ist. Danach folgen auf fols. 55 bis 69 Zeichnungen, die teilweise noch in Originalen Leonardos erhalten, teilweise aber verloren sind und die Oberflächenmaße des bewegten menschlichen Körpers zeigen. ${ }^{7}$ Dazwischen finden sich auf einigen Blättern verstreute Skizzen zum Problem der Proportionen im Verhältnis zur Zentralperspektive.

Der aus 128 Blättern bestehende Codex Huygens ist eine nicht mehr vollständige Sammlung verschiedenster Gebiete, die, neben den genannten Proportions- und Bewegungsstudien, auch einige nach Leonardo angefertigte Durchzeichnungen zu den Proportionen der Pferde sowie ausführliche Anweisungen zur Perspektive enthält. ${ }^{8}$ Der Codex wurde benannt nach Constantin Huygens, der jenen "livre in quarto escrit et dessigné de Leonardo da Vinci" im Jahr 1690 von der Witwe des 1677 in London verstorbenen Malers Remy van Leemput gekauft hatte. ${ }^{9}$ Leemput selbst, der eine umfangreiche Sammlung von Stichen und Zeichnungen besaß, mag das Huygensmanuskript durch seine in Rom tätigen Söhne erhalten haben. ${ }^{10}$ Kurze Zeit nach dem Verkauf des Codex Huygens aus dem Nachlaß Leemputs veröffentlichte Edward Cooper in London einige Auszüge, die sogenannten "Cooper engravings". ${ }^{11}$ Nach seiner Entdeckung im 17. und seiner teilweisen Publikation im 18. Jahrhundert taucht der Codex Huygens erst wieder 1915 auf, beschrieben durch A. W. M. Mensing, der das damals in seiner Sammlung befindliche Konvolut für wenig leonardesk hielt und eher michelangeleske Züge darin sah. ${ }^{12}$ Gegen Ende der dreißiger Jahre schließlich erwarb die Pierpont Morgan Library in New York das Manuskript.

Die Geschichte des Codex Huygens vor seinem Ankauf durch Constantin Huygens ist bis heute weitgehend ungeklärt. Gian Paolo Lomazzo bezieht sich gegen Ende des 16. Jahrhunderts möglicherweise auf die Studien der Bewegungsabläufe ${ }^{13}$ und auf Darstellungen verkürzter Figuren im Huygensmanuskript. ${ }^{14}$ Vielleicht existierten auch Verbindungen zu einem Proportionstraktat Enea Salmeggias (ca. 1565/70-1626), denn Francesco Maria Tassi (1710-1782) beschreibt in seinen 1793 posthum erschienenen "Vite de' pittori, scultori e architetti Bergamaschi" eine Kladde (scartafaccio) aus dem Besitz Salmeggias, deren Inhalt jenem Codex teilweise ähnelt. ${ }^{15}$ Doch sind die Beschreibungen Tassis letztlich zu ungenau für ein gesichertes Urteil. Gesichert hingegen scheint die Autorschaft Carlo Urbinos ${ }^{16}$, der den Codex in den 60er Jahren des Cinquecento aus Material zusammenstellte, das zu einem bislang ungeklärten Umfang auf Zeichnungen und Notizen beruht, die direkt oder indirekt von Leonardo stammen. Neben den eindeutig nach Originalen Leonardos kopierten Proportionszeichnungen und den anderen, oben genannten Studien sind vor allem die ersten dreißig Blätter interessant, von denen 26 Seiten geometrisch schematisierte Bewegungen des 
menschlichen Körpers zeigen. Die oft in Kreise einbeschriebenen Darstellungen erscheinen allerdings - wie bereits Mensing bemerkte - stilistisch wenig leonardesk, und ihre auffallende Geometrisierung weist eine mehr oder weniger offensichtliche Verwandtschaft mit lediglich einer einzigen Leonardozeichnung auf, nämlich mit seiner bekannten und heute in Venedig (Gallerie dell'Accademia) befindlichen Darstellung der Proportionsfigur Vitruvs, dem sogenannten "homo vitruvianus" (Abb. 1). ${ }^{17}$ Diese Verwandtschaft ist allerdings der einzige verläßliche Ausgangspunkt ${ }^{18}$, denn darüber hinaus hat man bisher keine schlüssigen Beweise für weitere Verbindungen zwischen den Bewegungsstudien des Codex Huygens und eventuellen Vorbildern Leonardos beizubringen vermocht; vielmehr sind diese Verbindungen ${ }^{19}$ kaum nachvollziehbar und für eine glaubwürdige Argumentation unbrauchbar. Trotzdem ist oft erfolglos über die originale Vorlage des Codex gemutmaßt ${ }^{20}$, über seinen Autor gestritten und über seine Verbindungen zum Werk Leonardos spekuliert worden. ${ }^{21}$

Weder zu eindeutigen noch unwidersprochenen Ergebnissen sind auch die bisherigen Versuche gelangt, plausible Datierungen für die leonardesken Vorlagen des im Codex Huygens erhaltenen Materials zu finden. So hatte Irma Richter als Datum "post quem non" das von Luca Pacioli (s.u.) erwähnte Jahr 1498 vorgeschlagen ${ }^{22}$, wohingegen Carlo Pedretti zunächst Leonardos Spätphase als chronologischen Bezugspunkt annahm ${ }^{23}$, später aber - aufgrund stilistischer Kriterien - sowohl mit der von Irma Richter vorgeschlagenen Datierung (1498) symphatisierte, als auch den Zeitraum zwischen 1505 bis 1510 ins Auge faßte. ${ }^{24}$ Kürzlich noch wurde argumentiert, der Codex Huygens erinnere an Leonardos "zeichnende Hand" der Zeit um 1505. ${ }^{25}$

Aufgrund der genannten Widersprüche und Probleme bedarf es, um die Bewegungsstudien des Codex Huygens tatsächlich und überzeugend mit Leonardo verbinden zu können, der weitergehenden und grundsätzlichen Erörterung, ob jene geometrisierenden Bewegungsstudien schon zu Lebzeiten Leonardos existierten und ob solche Studien in einem nennenswerten Umfang von Leonardo selbst stammen konnten. Zur Klärung dieser Frage stehen zur Verfügung: erstens frühe Darstellungen ähnlicher Proportionsstudien, deren leonardesker Ursprung keinem Zweifel unterliegt; zweitens Schriftquellen, in denen solche Studien mit hinreichender Genauigkeit beschrieben werden. Es folgt zunächst die Analyse der Bildquellen.

Zwischen den erst in der zweiten Jahrhunderthälfte kompilierten Bewegungsdarstellungen des Codex Huygens und ähnlichen Studien des bereits 1519 verstorbenen Leonardo besteht eine chronologische Lücke; diese Lücke war schon immer problematisch, denn sie ließ den Schluß zu, daß die im Huygensmanuskript erhaltenen Studien lediglich einen sehr geringen leonardesken Kern haben und im übrigen das Werk nicht identifizierter Epigonen seien. Um die Bewegungslehre des Codex Huygens also chronologisch und damit auch inhaltlich an das gesicherte Oeuvre Leonardos zu rücken, müßte die besagte Lücke geschlossen werden. Sie verkleinerte sich zunächst um wenige Jahre, als Carlo Pedretti eine dem Girolamo Figino zugeschriebene Zeichnung aus Oxford (Christ 
Church) publizierte, die als Verbindungsglied zu den Originalen Leonardos gelten kann und deren Wasserzeichen auf eine Datierung vor 1555 schließen läßt (Abb. 2) ${ }^{26}$ Allerdings belegen das sogenannte "Oxford sheet" und auch andere Quellen dieses Zeitraums wie der 1562 publizierte "Discorso generale" Antonio Maria Venustis ${ }^{27}$ oder der 1553 veröffentlichte "Trattato di scientia d'arme" Camillo Agrippas lediglich ${ }^{28}$, daß bestimmtes, hier als leonardesk eingeschätztes und ab den 60er Jahren in den Codex Huygens kompiliertes Material in der Mitte des 16. Jahrhunderts einem größeren Pesonenkreis bekannt war. Es fehlt damit also immer noch die Schließung der "chronologischen Lücke."

Erst durch die Analyse einer weiteren Quelle wurde kürzlich das Material der Bewegungsstudien im Codex Huygens um zwei Jahrzehnte näher an die Lebenszeit Leonardos gerückt. Und zwar finden sich in den 1533 zuerst vollständig erschienenen, aber bereits 1531 druckfertigen "De occulta philosophia libri tres" Agrippa von Nettesheims einige Holzschnitte und Notizen, die nicht nur enge Beziehungen zum Codex Huygens aufweisen, sondern auch zu Leonardos Zeichnung Vitruvischer Proportionen in der Accademia zu Venedig (Abb. 1). ${ }^{29}$ Aus einer detaillierten Analyse der einzelnen Zusammenhänge folgt, daß Agrippas Holzschnitte (Abb. 3a-b) und auch seine dazugehörigen Erläuterungen sowohl eine Beziehung zu Leonardos Zeichnung Vitruvischer Proportionen als auch zu fol. 7 des Codex Huygens haben (Abb. 4). Die Verbindungen, die auch zum "Oxford sheet" Girolamo Figinos (Abb. 2) bestehen, ergeben sich vor allem aus übereinstimmenden geometrischen Konstruktionen und aus teilweise exakten Entsprechungen im Bereich detaillierter Proportionsangaben. Das Material Agrippas verbindet also sowohl zeitlich wie inhaltlich die Studien Leonardos mit den Kompilationen des Codex Huygens. ${ }^{30}$

Eine weitere zeitliche Annäherung zwischen dem Codex Huygens und den Studien Leonardos um zunächst zwei Jahre ermöglicht der 1529 erschienene "Champfleury" des Pariser Druckers Gefroy Tory. ${ }^{31}$ In diesem Buch finden sich zahlreiche von Vitruv inspirierte Proportionsfiguren, die zum größten Teil auf die Vitruvausgabe Cesare Cesarianos zurückgehen ${ }^{32}$, in einem Fall aber leonardesken Ursprungs gewesen sein müssen. Torys Holzschnitte auf fol. 46v seines "Champfleury" (Abb. 5) zeigen Darstellungen eines in zwei Kreisen und einem Quadrat eingeschriebenen jungen Mannes, die, wie auch die Holzschnitte Agrippas und das Blatt aus Oxford, als ein "missing link" zwischen ähnlichen Zeichnungen im Codex Huygens und den Proportionsstudien Leonardos gelten können.

Veranschaulicht wird im "Champfleury" ein bestimmtes Schema zweier Kreise, eines großen und eines kleineren, denen jene Proportionsfigur eingeschrieben ist, die Vitruv beschrieben und Leonardo da Vinci mit seiner heute in Venedig befindlichen Zeichnung illustriert hat. Leonardos besondere Lösung des Problems, wie zwei Figuren, der "homo ad quadratum" und der "homo ad circulum", in einer Zeichnung zusammen dargestellt werden können, muß neben Agrippa und Girolamo 
Figino auch Tory bekannt gewesen sein, denn von keinem anderen Künstler ist eine in diesem Maße physiologisch überzeugende Darstellung des sogenannten "homo vitruvianus" überliefert: Vitruv schreibt, daß ein wohlgestalteter Mensch bei ausgestreckten Armen sein Zentrum im Nabel habe, so daß man eine kreisförmige Linie um ihn schlagen könne. Gleichzeitig veranschauliche ein Mann mit ausgebreiteten Armen jene Flächen, die nach dem Winkelmaß des Architekten quadratisch seien. $^{33}$ Die Vereinigung beider Figuren in einer einzigen Darstellung macht dann Schwierigkeiten, wenn man, wie die Künstler nach Leonardo, den "homo vitruvianus" in Kreise und Quadrate einschreibt, die in einem geometrischen Verhältnis zueinander stehen. Dieses geometrische Verhältnis, die "Quadratur", deren Bedeutung vor allem für den Entwurf von Kleinformen in der mittelalterlichen Architektur nachgewiesen wurde ${ }^{34}$, ignoriert Leonardo vollständig. Stattdessen orientierte er sich in seiner Dimensionierung des Quadrats an den Ergebnissen anthropometrischer Untersuchungen, die er zwischen 1489 und 1490 an zwei wohlproportionierten jungen Männern vorgenommen hatte. $^{35}$ Der umschreibende Kreis steht dann in keinem geometrischen Verhältnis mehr zum Quadrat, sondern erhält seinen Radius aufgrund jener Entfernung, die zwischen dem Nabel und den ausgestreckten, bis auf Kopfhöhe erhobenen Händen besteht. In Leonardos Zeichnung des "homo vitruvianus" fehlt der kleinere Kreis, da er dort keine Funktion gehabt hätte, doch bei Tory und im "Oxford sheet", besonders aber im Codex Huygens fungiert er in seinem Verhältnis zum großen Kreis als geometrische Schematisierung der Bewegung des menschlichen Körpers. ${ }^{36}$ Diese Schematisierung - variiert sowohl bei Tory und im "Oxford Sheet" als auch im Codex Huygens - folgt direkt aus Leonardos Zeichnung des "homo vitruvianus".

Mit dem "Champfleury", dessen Holzschnitte zwischen Leonardos Vitruvstudie, dem "Oxford sheet" und dem Codex Huygens vermitteln, ist als ein weiteres Datum zunächst erst das Jahr 1529 gesichert, doch da Tory seine Quelle selbst angibt, kann man die chronologische Lücke noch erheblich verkleinern: Im Text erklärt er, jene Darstellungen des Mannes im Kreis von seinem Freund Jean Perréal erhalten zu haben. Perréal (ca. 1455-1530) stand zu jener Zeit in den Diensten Franz I. und hatte vorher in den verschiedensten Funktionen die französischen Könige Karl VIII. und Ludwig XII. nach Italien begleitet, wo er auch mit Leonardo zusammengetroffen ist. ${ }^{37}$ Ein quasi symbolisches Dokument für dieses Ereignis findet sich in einer auf ca. 1509 datierten Handschrift der Bibliothèque Nationale zu Paris. Dort zeigt eine Initiale die beiden Porträts Leonardo da Vincis und Jean Perréals. $^{38}$ Die mehrfachen Italienaufenthalte Jean Perréals lagen teilweise schon vor dem Jahr 1509, und Leonardo selbst erwähnt den französischen Künstler folgendermaßen: "piglia da Gian di Paris il modo de colorire a secco [...]." ${ }^{39}$ Diesem Hinweis kann man entnehmen, daß Leonardo den Maler Jean Perréal vor Abschluß der Arbeiten am Abendmahl in S. Maria delle Grazie in Mailand, also vor dem Sommer 1497, um Anweisungen zur a-secco-Malerei gebeten hat. Sogar ein Datum vor 1497 ist möglich, da Leonardo die besagten Auskünfte über die Maltechnik Perréals bereits 
zu Beginn der Arbeiten am Abendmahl hätte eingeholt haben können, d.h. in der Zeit zwischen 1495 und 1496. Jean Perréal konnte also schon um 1497 oder wenig früher jene geometrisierenden und an Vitruv orientierten Proportionsstudien kopiert haben, die heute durch Gefroy Tory überliefert sind.

Die Tatsache, daß Jean Perréal und Agrippa von Nettesheim identische Bewegungsstudien leonardesken Ursprungs sahen, ist möglicherweise kein Zufall, denn zwischen beiden Männern, deren Bekanntschaft über gemeinsame alchimistische Interessen begonnen haben mag ${ }^{40}$, hat es nachweislich enge Verbindungen gegeben. Bereits 1509 erwähnt Agrippa den französischen Hofmaler in einem Brief aus Dole, 1524 läßt er ihn aus Fribourg grüßen, und im April 1527 hofft er "seinen Freund" in Lyon bald wiedersehen zu können. ${ }^{41}$ Nur sechs Monate später, im September desselben Jahres, stellt Agrippa die endgültige Edition seiner "Occulta philosophia" in Aussicht. ${ }^{42}$ Aufgrund dieser Situation und angesichts der inhaltlichen Übereinstimmungen zwischen der bei Tory abgedruckten leonardesken Proportionsfigur Perréals einerseits und ähnlichen Darstellungen in der "Occulta philosophia" andererseits, könnte man schließlich die These wagen, daß Jean Perréal die aus dem theoretischen Oeuvre Leonardos stammenden Entwürfe nicht nur nach Frankreich mitgebracht, sondern auch seinem Freund Agrippa von Nettesheim zur weiteren Benutzung überlassen hatte. Ausgehend von den Ideen Leonardos, wäre Perréal also der Illustrator der "Occulta philosophia".

Mit Perréals Rezeption leonardesker Bewegungsdarstellungen ist die chronologische Lücke zwischen dem Codex Huygens und den Proportionsstudien Leonardos (also zwischen 1560 und 1490) zwar geschlossen, doch fehlt in den bisher genannten Belegen der Name Leonardos. Dieses Manko kompensieren jene Quellen, deren bloße Beschreibungen auf nennenswerte, aber heute verlorene Proportions- und Bewegungsstudien Leonardos schließen lassen. Mit dem oben ermittelten Datum, 1497, bekommt die früheste dieser Quellen, Luca Paciolis "De divina proportione", eine ernstzunehmende Bedeutung, denn Pacioli erwähnt in seiner auf den 9. Februar 1498 datierten Widmung einen von Leonardo bereits abgeschlossenen "libro di pittura e movimenti humani". ${ }^{43}$ Bisher hat man immer auf Paciolis notorischen Hang zur Ungenauigkeit und damit auf die Möglichkeit hinweisen können, daß er lediglich größere Mengen von Zeichnungen Leonardos für eine bereits fertige Abhandlung gehalten hatte. Da aber Jean Perréal um etwa dieselbe Zeit ebenfalls Bewegungsstudien sah, die eine eindeutige Beziehung sowohl zu Leonardos Vitruvzeichnung in Venedig als auch zu weitaus umfangreicherem Material im Codex Huygens haben, gewinnt Paciolis Aussage ein hinreichendes Maß an Glaubwürdigkeit. Wenn man also die Proportionsfigur von der Hand Perréals, die Bewegungsstudien im Codex Huygens und die Angaben Paciolis in einem Zusammenhang sieht, gibt es Gründe zu der Annahme, daß Leonardo vor 1497/1498 tatsächlich Studien zu einem vorläufigen Abschluß gebracht hatte, deren ursprüngliche Gestalt mit den Zeichnungen des Codex Huygens eng verwandt, wenn nicht gar identisch war. 
Vor dem Hintergrund der bisherigen Ausführungen erhält auch eine andere Quelle größeres Gewicht, nämlich Federigo Zuccaros 1607 erschienene "Idea de' pittori, scultori ed architetti". Ihr Autor schreibt anläßlich eines proportionstheoretischen Diskurses folgendes über die Künstler Albrecht Dürer und Leonardo da Vinci:

"Gleichermaßen unergiebig (di poco frutto) und von geringem Wert war eine weitere Lehre, die ein anderer sonst tüchtiger Mann unseres Metiers hinterließ, entworfen zusammen mit spiegelbildlicher Schrift, aber wunderlich auch er, rein mathematische Regeln zu hinterlassen, um die Figur mit senkrechten Linien, mit dem Winkelmaß und mit Zirkeln zu bewegen und zu drehen. ${ }^{44}$

Die Beschreibung der mit Winkelmaß, Zirkel und senkrechten Linien gestalteten Bewegungsstudien paßt sehr genau zu jenen Darstellungen, die auf den ersten 30 Blättern des Codex Huygens zusammengetragen sind. Zuccaro kann sich aus folgenden Gründen auf jene Originale Leonardos bezogen haben, die der Kompilation der Kopien im Codex Huygens zugrundelagen: Erstens ist ausdrücklich von Spiegelschrift die Rede (scritti alla rouerscia), es dürfte also kaum der in normaler Schrift geschriebene Codex Huygens gemeint gewesen sein, und auch ein anderer Künstler kommt aufgrund der Spiegelschrift nicht infrage. Zweitens, sowohl die aus den Originalen Leonardos erhaltenen als auch die im Codex Urbinas überlieferten Studien zur menschlichen Proportion passen nicht auf Zuccaros Beschreibung; es bleibt als einzige Möglichkeit also nur verlorenes und mit höchster Wahrscheinlichkeit in den Codex Huygens kopiertes Material. Eben dieses Material müssen auch Pacioli, Perréal, Agrippa von Nettesheim und Künstler wie Girolamo Figino vor Augen gehabt haben, denn die bisher genannten Details, Daten und Übereinstimmungen schließen mit an Sicherheit grenzender Wahrscheinlichkeit die Möglichkeit aus, daß der originale Kern der im Codex Huygens erhaltenen Bewegungsstudien ursprünglich nicht von Leonardo da Vinci stammte. Man kann also - erstmals nach Auffindung der Codices Madrid ${ }^{45}$ - das theoretische Schaffen Leonardos unter dem Gesichtspunkt neuen Materials analysieren.

II.

Unter Berücksichtigung des im Codex Huygens überlieferten Materials können die Proportions- und Bewegungsstudien Leonardos grob in drei verschiedene Perioden unterteilt werden. Die früheste Phase, die in den Manuskripten vor allem hinsichtlich der Zeichnungen am besten dokumentiert ist, begann etwa 1483 mit Leonardos Ankunft in Mailand und konzentriert sich besonders auf die Jahre 1489 bis $1493 .{ }^{46}$ Unmittelbar daran anschließend hat es einen weiteren, im Detail noch zu erörternden Schwerpunkt gegeben, der gegen 1498 beendet gewesen sein muß (s.u.). Eine völlig neue, dritte Phase der Proportions- und Bewegungsstudien Leonardos kann man schließlich nach der Jahrhun- 
dertwende, etwa zwischen den Jahren 1505 und 1515 feststellenen.

Der zweite Abschnitt der Proportionsstudien Leonardos ist bisher am wenigsten beachtet worden, da hierzu kein originales Material vorliegt. Dieses Manko kann nun teilweise ausgeglichen werden, denn die oben eruierten chronologisch relevanten Hinweise aus den Quellen, namentlich aber die durch Jean Perréals Italienaufenthalt fixierten Daten erlauben eine exakte zeitliche Einordnung des Materials aus dem Codex Huygens in Leonardos Oeuvre der 90er Jahre des 15. Jahrhunderts. Jene Darstellung eines Mannes im Kreis und Quadrat, die Tory 1529 publizierte und Perréal bereits vor 1497 im Besitz Leonardos sah, ist so eng mit den geometrisierenden Bewegungsstudien des Codex Huygens verwandt, daß deren originale Vorlagen zu demselben Zeitpunkt - wenn man der Aussage Paciolis Glauben schenkt - vollendet gewesen sein müssen. Auch der Beginn der geometrisierenden Bewegungsstudien läßt sich mit hinreichender Genauigkeit bestimmen, denn die Verbindung dieser Studien zu der 1490 entstandenen Zeichnung Vitruvischer Proportionen in Venedig ist offensichtlich und bis in Details hinein nachweisbar. ${ }^{47}$ Daraus ergibt sich insgesamt ein Zeitraum von acht Jahren, 1490 bis 1497, der für die Entstehung der in den Codex Huygens kompilierten Bewegungsdarstellungen Leonardos infrage kommt. Dieser Zeitraum schloß (mit einer zeitlichen Überschneidung von 4 Jahren, 1490-1493) chronologisch an die frühesten Proportionsstudien Leonardos an, die 1490 in der Zeichnung des "homo vitruvianus" ihren prägnanten Ausdruck fanden. Die Darstellung der Proportionsfigur Vitruvs war also sowohl der Ausgangspunkt jener verlorenen Studien, die nur mittelbar aus dem Champfleury Torys, der Occulta Philosophia Agrippas, aus dem "Oxford sheet" und aus dem Codex Huygens bekannt sind, als auch das unmittelbare Produkt vorangegangener anthropometrischer Studien, die aufgrund ihrer Homogenität nicht vor das Jahr 1489 zurückgehen und aufgrund anderer chronologisch relevanter Indizien nicht nach 1493 entstanden sein können (Abb. 9-14).

Die Entstehungsgeschichte der an zentraler Stelle plazierten Vitruvzeichnung - und damit ihre Verbindung zur ersten Phase der Proportionsstudien - läßt sich aus einigen Blättern rekonstruieren, die alle um $1490 \mathrm{zu}$ datieren sind: Anthropometrische Ergebnisse, die Leonardo aufgrund der Vermessung zweier Modelle namens "Trezzo" und "Caravaggio" erzielte, hielt er zunächst in einer Reihe von Zeichnungen fest (Abb. 9-10), um sie danach in das bei Vitruv beschriebene Proportionssystem einzuarbeiten (Abb. 10-14). Daraus resultierte nicht nur die Darstellung des "homo vitruvianus" (Abb. 1), sondern mittelbar auch jenes Konzept geometrisierter Bewegung, das heute nur noch durch die im "Oxford sheet" (Abb. 2) sowie bei Tory, Agrippa (Abb. 5 und 3a-e) und im Codex Huygens (Abb. 4, 6, 7, 8) überlieferten sowie bei Pacioli und Zuccaro erwähnten Darstellungen dokumentiert ist. $^{48}$

Die beiden eng miteinander zusammenhängenden ersten Phasen der Proportions- und Bewegungsstudien Leonardos könnte man unter Berücksichtigung ihrer wichtigsten Prinzipien mit 
"anthropometrisch" (1489-1493) und "geometrisch" (1493-1498) bezeichnen. Im Gegensatz dazu steht die spätere Schaffensperiode, deren Bewegungsdarstellungen nach 1500, vorwiegend sogar nach 1505 entstanden sind und heute zum größten Teil aus Francesco Melzis Kompilationen und Nachzeichnungen im Codex Urbinas rekonstruiert werden müssen. Die Unterschiede zwischen den beiden früheren Abschnitten und der spätesten Periode zeugen von einer völlig neuen Aufnahme der menschlichen Körperbewegung; an die Stelle einer aus der Anthropometrie entwickelten und danach an der Geometrie orientierten Analyse von Bewegungsabläufen tritt nach der Jahrhundertwende die skizzenhafte und unschematische Entwicklung impulsiver Bewegungen. Es scheinen sich nicht nur neue Ideale körperlicher Schönheit und stilistischer Modellierung durchgesetzt, sondern auch andersartige Fragestellungen ergeben zu haben: Während die besonders um das Jahr 1490 betriebene Anthropometrie und die anschließenden Bewegungsstudien der 90er Jahre vorwiegend auf der Grundlage einer anthropometrisch und geometrisch fundierten Meßbarkeit basierten, tauchen nach 1500 zunehmend Bewegungen auf, die inhaltlich motiviert sind und kaum noch meßbaren Prinzipien gehorchen. D.h. nicht mehr abstrakte Vermessung und geometrische Schematisierung, sondern konkrete Motive wie Speerwerfen, Lasten-schleppen, Sich-umwenden, Stoßen, Waffen-schleudern oder Kämpfen bildeten nach 1500 den Kern von Leonardos Auseinandersetzung mit der Bewegung (Abb. 15a-f). ${ }^{49}$ Die nunmehr reduzierte Relevanz der Meßbarkeit wird durch Leonardos unterschiedliche Behandlung des körperlichen Gleichgewichts veranschaulicht, das er noch um 1492 mit Hilfe des klassischen Kontraposts und in kommensurablen Äquivalenten auszudrücken versuchte. ${ }^{50}$ Später jedoch entschied er sich für eine freiere Skizzierung, so daß die Balance keinem Prinzip von Meßbarkeit mehr unterworfen war. ${ }^{51}$ Außerdem scheint er nach der Jahrhundertwende die besondere Bedeutung des Gleichgewichts so hoch eingeschätzt zu haben, daß er zwischen 1500 und 1505 der Bearbeitung dieses Gegenstands ein ganzes Buch widmen wollte. ${ }^{52}$

III.

Sowohl die oben vollzogene Anbindung der im Codex Huygens und in anderen Quellen überlieferten Bewegungsdarstellungen an das theoretische Oeuvre Leonardos als auch deren chronologische Einordnung in die Gesamtheit seiner Proportionsstudien ermöglichen eine genauere Periodisierung dieser Studien. Die in der ersten Phase (1489-1493) inaugurierte Vermessbarkeit des menschlichen Körpers (Anthropometrie) fand eine konsequente und unmittelbare Fortsetzung in dem Versuch, die Bewegungen zu geometrisieren (1490-1498), denn die Geometrie (ursprünglich die Wissenschaft von der Terrainvermessung ${ }^{53}$ ) repräsentierte gegenüber der vorher ausgeführten Anthropometrie die höhere Wissenschaftlichkeit der "soma verità" ${ }^{54}$, die nicht nur eine rational verifizierbare Schematisierung der Bewegungen gewährleisten, sondern auch ein visuell kontrollierbares Prinzip der Kommensurabilität garantieren sollte. Nach seiner Abreise aus Mailand geriet Leonardo durch den er- 
neuten Florentiner Aufenthalt in ein anderes künstlerisches Ambiente: In der Konkurrenz mit Michelangelo, anläßlich des Auftrags für die Signoria, begann Leonardo die dritte, von den beiden vorhergehenden so verschiedene Phase seiner Proportions- und Bewegungsstudien. Nur wenig später vertiefte er sein Interesse an der menschlichen Figur, das bis dahin überwiegend den Oberflächenmaßen gegolten hatte: mit der Wiederaufnahme und Intensivierung seiner Anatomiestudien ging Leonardo nun buchstäblich unter die Haut des Körpers. Damit konzentrierte er seine immense wissenschaftliche Neugier immer mehr auf Gebiete, deren unmittelbare künstlerische Relevanz kaum noch vermittelbar gewesen wäre; gleichzeitig ermöglichte die Bindung seines wissenschaftlichen Ehrgeizes an die Anatomie eine neuartige Aufnahme der Bewegungsstudien, so daß die im Codex Urbinas erhaltenen Figuren wie befreit von den Ansprüchen der Wissenschaftlichkeit erscheinen. Zum Zeitpunkt dieser "figürlichen Befreiung" befand sich Leonardo nicht mehr am Hof der Sforza; für verschiedene Auftraggeber und mit diversen Aufträgen war er nun in unterschiedlichen Gegenden Italiens tätig und sah sich anderen Anregungen ausgesetzt als während der ersten Mailänder Periode.

Die unterschiedlichen Bewegungsstudien, entwickelt sowohl aus Anthropometrie und Geometrie als auch aus spontan wahrgenommenen Motiven $^{55}$, zeugen von einem außerordentlich starken Interesse an der Bewegung, das sich praktisch durch das gesamte Oeuvre Leonardos zieht. Daher tauchen in seinem Werk zu verschiedensten Zeiten Motive auf, deren figürliche Disposition den Bewegungsdarstellungen des Codex Huygens verwandt ist. ${ }^{56}$ Dies gilt sowohl für einige schematische Parallelen (am Beispiel eines Baumes und einer Hand) ${ }^{57}$ als auch für ein besonderes Charakteristikum der Kompositionen Leonardos: Die Entwicklung von Bewegungen aus einer Figur, die Ableitung verschieden bewegter Figuren aus den bewegten Varianten einer einzelnen Figur. ${ }^{58}$ Das vielleicht beste Beispiel hierfür ist die Darstellung der Hl. Anna Selbdritt (Paris, Louvre; Abb. 16), deren endgültige Fassung wohl von ca. 1508/1510 stammt, aber auch noch Bewegungskonzepte der späten 90er Jahre des Quattrocento berücksichtigt ${ }^{59}$ : Die Sequenz der Figuren entwickelt sich, als ob Anna und Maria denselben Körper hätten (auch ikonographisch eine interessante Assoziation), und die inhaltlich schwer zu rechtfertigende Gleichaltrigkeit der beiden Frauen bestätigt diesen Eindruck einer körperlichen Einheit, deren figürlicher Abfolge sogar der mit dem Lamm spielende Christus anzugehören scheint. Die drei sichtbaren Füße von Maria und Anna schließlich folgen einer rhythmischen Gleichmäßigkeit, die den kinematographischen Bewegungssequenzen des Codex Huygens eng verwandt ist und schon fast zu künstlich wirkt. Den Eindruck eines künstlichen oder kunsttheoretischen "capriccio" macht auch die Anordnung des rechten Fußes der Maria, rechts neben dem linken Fuß der Anna: es scheint auf den ersten Blick so, als ob Maria ihre Beine auf sehr merkwürdige Art und Weise übereinander geschlagen habe. Erst aus der näheren Beobachtung der ineinander bewegten Figuren entwirrt sich die figürliche Disposition, so daß der Betrachter aus dem Nach- 
vollzug der Bewegungen die eigentliche Komposition erkennt. Eben dieses Kompositionsprinzip läßt sich bei kaum einem anderen Künstler jener Epoche in vergleichbarem Maße aus kunsttheoretischen Studien entwickeln.

Aufnahmen: 1. Venedig, Galleria dell'Accademia; 2. Oxford, Christ Church Galery; 3a-b Agrippa von Nettesheim, De occulta philosophia, Köln 1533; 4 und 6-8. New York, Pierpont Morgan Library; 5. G. Tory, Champfleury, Paris 1529; 9-14. The Royal Library at Windsor Castle (by gracious permission of Her Majesty the Queen); 15a-f. Rom, Biblioteca Apostolica Vaticana; 16. Paris, Louvre.

Bildunterschriften

1. Leonardo, Proportionszeichnung nach Vitruv, Accademia, Venedig

2. Girolamo Figino (?), Proportionsfigur, Oxford, Christ Church.

3a. Holzschnitt aus: Agrippa von Nettesheim, De occulta philosophia, Köln 1533, fol. 165.

3b. Holzschnitt aus: Agrippa von Nettesheim, De occulta philosophia, Köln 1533, fol. 163.

4. Codex Huygens, Pierpont Morgan Library, New York, fol. 7.

5. G. Tory, Champfleury, fol. 46v

6. Codex Huygens, Pierpont Morgan Library, New York, fol. 5.

7. Codex Huygens, Pierpont Morgan Library, New York, fol. 12.

8. Codex Huygens, Pierpont Morgan Library, New York, fol. 15.

9. Leonardo, Proportionszeichnungen, Windsor Castle, W.19136-139v.

10. Leonardo, Proportionszeichnungen, Windsor Castle, W.19136-139r.

11. Leonardo, Proportionszeichnungen, Windsor Castle, W.19130v.

12. Leonardo, Proportionszeichnungen, Windsor Castle, W.19130r.

13. Leonardo, Proportionszeichnungen, Windsor Castle, W.19131r.

14. Leonardo, Proportionszeichnungen, Windsor Castle, W.19132r.

15a. Codex Urbinas, Biblioteca Apostolica Vaticana, Rom, fol. 106r.

15b. Codex Urbinas, Biblioteca Apostolica Vaticana, Rom, fol. 112v.

15c. Codex Urbinas, Biblioteca Apostolica Vaticana, Rom, fol. 119v.

15d. Codex Urbinas, Biblioteca Apostolica Vaticana, Rom, fol. 120r.

15e. Codex Urbinas, Biblioteca Apostolica Vaticana, Rom, fol. 128r.

15f. Codex Urbinas, Biblioteca Apostolica Vaticana, Rom, fol. 128v.

16. Leonardo, Anna Selbdritt, Paris, Louvre. 


\section{Anmerkungen}

1. E. Panofsky: Die Proportionslehre als Abbild der Stilentwicklung, in: Monatshefte für Kunstwissenschaft 14,1921, 188-219. Vgl. danach z.B. G. Nicco Fasola (Hrsg.): Piero della Francesca. De prospectiva pingendi, Florenz 1942, 3-55; N. Speich: Die Proportionslehre des menschlichen Körpers, Phil. Diss., Zürich 1957; L. Salerno/ E. Battisti: Proportion, in: Encyclopedia of World Art, Bd. 11, New York etc. 1966, Sp. 716-738; F. Borsi, Per una storia della teoria delle proporzioni, Florenz 1967; L. Moya Blanco: Notas sobre las proporciones del cuerpo humano segun Vitruvio y San Agustin, in: Academia. Boletin de la Real Academia de bellas Artes de San Fernando 46,1978, 39-60.

2. Vgl. H. Klaiber: Leonardostudien, Straßburg 1907, 100-109; G. Favaro: Il canone di Leonardo, in: Atti del Reale istituto Veneto di scienze, lettere ed arti 77,1917, 167-227; A. Pazzini (Hrsg.): Leonardo da Vinci. Il trattato della anatomia, 3 Bde., Rom 1963, Bd. 1, 61-95; H. Ost: Leonardo-Studien, Berlin/ New York 1975, $12-29$.

3. Vgl. J. Giesen: Dürers Proportionsstudien im Rahmen der allgemeinen Proportionsentwicklung, Bonn 1930; R. Keil: Zu Dürers frühen Proportionszeichnungen des menschlichen Körpers, in: Pantheon 43,1985, 54-61.

4. Vgl einige Ansätze bei z. B. L. Fusco: The Nude as Protagonist: Pollaiuolo's Figural Style Explicated by Leonardo's Study of Static Anatomy, Movement and Functional Anatomy, Phil. Diss., New York 1978; dies.: The Use of Sculptural Models by Painters in Fifteenth-Century Italy, in: The Art Bulletin 64,1982, 175-194.

5. Vgl. G. Calvi: I Manoscritti di Leonardo da Vinci dal punto di vista cronologico, storico e biografico, Bologna 1925 (jetzt benutzbar in einer neuen, 1982 erschienenen und von A. Marinoni besorgten Ausgabe); J. P. Richter: The Literary Works of Leonardo da Vinci, 2 Bde., 3. Aufl., London 1970, Bd. 2, 393-421. C. D. O'Malley/ J. B. de C. M. Saunders: Leonardo da Vinci on the Human Body, New York 1952, 33-35. A. Marinoni: I Manoscritti di Leonardo da Vinci e le loro edizioni, in: Leonardo da Vinci. Saggi e ricerche, Rom 1954, 229-274. C. Pedretti: Leonardo da Vinci on Painting. A Lost Book (Libro A), Los Angeles 1964. Ders.: The Literary Works of Leonardo da Vinci. Commentary, 2 Bde., Oxford 1977, Bd. 2, 393-402; K. D. Keele/ C. Pedretti: Leonardo da Vinci. Atlas der Anatomischen Studien in der Sammlung Ihrer Majestät Queen Elizabeth II in Windsor Castle, 3 Bde., London und Gütersloh 1978-1980, Bd. 1, xiii-xix; M. Kemp: Treasure Trove, in: Art Book Review 2,1983, Nr. 5, 26-28; K. Veltman: Studies on Leonardo da Vinci I. Linear Perspective and the Visual Dimensions of Science and Art, München 1986, 10. Über den ursprünglichen Umfang der Manuskripte gibt es allerdings verschiedene Meinungen; so geht C. Pedretti: Leonardo da Vinci. The Royal Palace at Romorantin, Cambridge (Mass.) 1970, 138, von 8000 erhaltenen und etwa doppelt so viel verlorenen Blättern aus.

Richter: Literary Works, $\S 797$ (W.19037v [81v]; diese Numerierung bezieht sich hier und im folgenden auf die Editionen von K. Clark/ C. Pedretti: The Drawings of Leonardo da Vinci in the Collection at Windsor Castle, 3Bde., 2. Aufl., London 1968-1969, und Keele/ Pedretti: Atlas der anatomischen Studien [in eckigen Klammern], zit. in Anm. 6).

7. Codex Huygens, fols. 53, 54, 55, 56, 57, 58, 59 (x), 60, 61 (x), 62 (x), 63 (x), 64, 65, 66, 67 (x), 68, 69 (x ?) - für die mit x bezeichneten folios existieren keine Originale Leonardos mehr. Weitere Blätter, fols. 70-86, sind Kopien nach Leonardos Studien der Proportionen des Pferdes. Vgl. E. Panofsky: The Codex Huygens and Leonardo da Vinci's Art Theory, London 1940, 51-58. 
8. Zum Codex Huygens allgemein vgl. Panofsky: The Codex Huygens; Pedretti: Commentary, Bd. 1, ix-x und 48-75. S. Marinelli: The Author of the Codex Huygens, in: Journal of the Warburg and Courtauld Institutes 44,1981, 214-220.

9. Vgl. Panofsky: Codex Huygens, 9-13; Pedretti: Commentary, Bd. 1, 48-49.

10. Vgl. Jakob Campo Weyerman: De Levens-Beschryvingen der Nederlandsche Konst-Schilders en KonstSchilderessen [...], 4 Bde., Dordrecht 1729-1769, Bd.4, 249-251; Thieme/Becker: Allgemeines Lexikon der bildenden Künstler, Bd. 22, Leipzig 1928, 544-545.

11. Vgl. C. Pedretti: Excerpts from the Codex Huygens published in London in 1720, in: Journal of the Warburg and Courtauld Institutes 28,1965, 336-338; Pedretti: Commentary, Bd. 1, 49-62.

12. A. W. M. Mensing: De Leonardo's van Constantijn Huygens den Zoon, in: Feest-Bundel Dr. Abraham Bredius, 2 Bde., Amsterdam o.J. [1915], Bd. 1, 186-190, Bd. 2, Tf. 80 und 81.

13. Gian Paolo Lomazzo: Idea del tempio della pittura, 5.8. (1590), in: Gian Paolo Lomazzo: Scritti sulle arti, Ed. R. P. Ciardi, 2 Bde., Florenz 1973-1975, Bd. 1, 269, könnte sich auf den Codex Huygens beziehen, was Marinelli: Author of the Codex Huygens, 215-216, jedoch bezweifelt; ein anderer Bezug, diesmal auf die Bewegungsfiguren, findet sich möglicherweise auch in Lomazzo: Trattato della pittura, 4.4. (1584), Ed. Ciardi, Bd. 2, 255-258.

14. Vgl. G. Bora (Hrsg.): Disegni di manieristi lombardi, Vicenza 1971, $42-47$ und Nr. 51-61.

15. Fransceso Maria Tassi: Vite de' pittori, scultori e architetti Bergamaschi, 2Bde., Bergamo 1793 (Nachdruck, Mailand 1969), Bd. 1, 217: "Da questi confusi frammenti si scorgono bellissime regole di prospettiva, con figure geometriche delineate, diversi utilissimi ricordi alla profession sua appartenenti, con molte graziose figurette di penna, secondo le diverse età dell'uomo, altre poste in iscorcio, di sotto in su, ed in altre difficile positure."

16. Vgl. U. Ruggeri: Carlo Urbini e il Codice Huygens, in: Critica d'arte 43,1978, H. 157-159, 167-176; Marinelli: Author of the Codex Huygens.

17. Vitruv: De architectura 3.1.; vgl. B. Reudenbach: In mensuram humani corporis. Zur Herkunft der Auslegung und Illustration von Vitruv III 1 im 15. und 16. Jahrhundert, in: Text und Bild. Aspekte des Zusammenwirkens zweier Künste in Mittelalter und früher Neuzeit, Ed. C. Meier und U. Ruberg, Wiesbaden 1980, 651-688; F. Zöllner: Vitruvs Proportionsfigur. Quellenkritische Studien zur Kunstliteratur im 15. und 16. Jahrhundert, Worms 1987.

18. Vgl. I. Richter: [Rez. Panofsky: Codex Huygens], in: The Art Bulletin, 23,1941, 335-338; Pedretti: Commentary, Bd. 1, 64-65.

19. Vgl. etwa W. 19136r [31r, Nr. 6, VI und VII]; W. 19035v [77v, Nr. II]; W.19038r [80r, Nr. II]; W.19113r [110r, Fig. vgl. Codex Huygens fol. 69]; W.19037v [81v].

20. So herrscht bis heute Verwirrung über angeblich noch im 18. Jahrhundert bekanntes Material Leonardos, das dem Codex Huygens zugrundegelegen habe und von dem vermeintlich Francesco Fontani in seiner 1792 erschienenen Ausgabe des Trattato della pittura berichtet. Irma Richter (Rez. zu Panofsky: The Codex 
Huygens) glaubte in dieser Edition einen Hinweis auf geometrisierende Bewegungsstudien Leonardos gefunden zu haben. Dies ist eindeutig ein Irrtum, denn ganz offensichtlich beschrieb Fontani ein Album von 9 Kupferstichen, das Edward Cooper um das Jahr 1720 nach Vorlage des Codex Huygens anfertigte. Die Möglichkeit, daß Fontani sich auf die Cooperschen Kupferstiche bezog, ist noch 1977 von Pedretti (Commentary, Bd. 1, 52) zurückgewiesen worden - wohl aufgrund einer zu flüchtigen Lektüre der TrattatoAusgabe Fontanis, denn dieser schreibt ausdrücklich von einem 9 Tafeln umfassenden Werk, das von einem Herrn Cooper in London herausgebracht worden sei: "Della sua grand' opera poi sulla Meccanica del corpo umano non se ne ha che un frammento pubblicato già in Londra molti anni sono dal Sig. Cooper [...] e consiste in nove tavole senza titolo [...]." Leonardo da Vinci: Trattato della pittura, Ed. F. Fontani, XVI, Anm. 26.

21. Vgl. die verschiedenen Ansichten bei: Pedretti: Libro A, 104-105 und 263-264; Pedretti: Commentary, Bd. 1, 70-75 P. Meller: Quello che Leonardo non ha scritto sulla figura umana: dall'uomo di Vitruvio alla Leda, in: Arte Lombarda, 67,1983, 117-133, 122; S. Braunfels-Esche: Aspekte der Bewegung. Umrisse von Leonardos Proportions- und Bewegungslehre, in: Leonardo da Vinci. Anatomie, Physiognomik, Proportion und Bewegung, Ed. O. Baur, B. Bott etc., 2 Bde., Köln 1984, Bd. 1, 84-117. Weitere Standpunkte, vor allem zum Autorenstreit, bei Marinelli: The Author of the Codex Huygens.

22. Vgl. I. Richter, [Rez. Panofsky, Codex Huygens].

23. Pedretti: Libro A, 17.

24. Pedretti: Commentary, Bd. 1, 65. Im Falle von mehr oder weniger freien Kopien und von Durchzeichnung ist natürlich eine stilkritische Argumtation grundsätzlich problematisch. Wenn man unbedingt stilistische Parallelen zwischen dem Codex Huygens und den Originalen Leonardos sehen möchte, könnte man diese vielleicht eher in einigen Blättern der 90er Jahre erkennen; vgl. etwa W.12603v [32v] oder W.19018r [41r].

25. Braunfels: Aspekte der Bewegung, 88; eine späte Datierung wie die von Braunfels ist um so unwahrscheinlicher, als etwa die drei im Codex Huygens enthaltenen Skelettstudien, fol. 2, im Vergleich zu Leonardos späten Skelettstudien von ca. 1510 (z. B. W. 19012r) qualitativ stark zurückfallen; vgl. O'Malley/ de C. M. Saunders: Human Body, Nr. 1, 40.

26. Pedretti: Commentary, Bd. 1, 68-75 und Tf. 10.

27. Antonio Maria Venusti: Discorso generale etc., Venedig 1562, c. 107-108; der Text auch bei Pedretti: Commentary, Bd. 1, 246-247.

28. Camillo Agrippa: Trattato di scientia d'arme, con un dialogo di filosofia, Rom 1553, c. IV-v, V-v (beide Codex Huygens, passim) und XIX-r (vgl. Codex Huygens, fol. 116, bei Panofsky: Codex Huygens, Abb. 68); vgl. auch C. L. Ragghianti: Scherma come geometria, in: Critica d'arte 22,1976, fasc. 148-149, 88-92; Marinelli: Author of the Codex Huygens, 218.

29. Agrippa von Nettesheim: De occulta philosophia, o.O. [Köln] 1533, fols. 161-166; vgl. Zöllner: Vitruvs Proportionsfigur, 196-198.

30. Vgl. F. Zöllner: Leonardo, Agrippa and the Codex Huygens, in: Journal of the Warburg and Courtauld Institutes 48,1985, 229-234. 
31. Gefroy Tory: Champfleury, Paris 1529, fol. 46v.

32. Vgl. Cesare Cesariano: Di Lucio Vitruuio Pollione de Architectura Libri Dece, Como 1521, fols. 49r und 50r.

33. Vitruv: De architectura 3.1.

34. Die sogenannte mittelalterliche Quadratur basiert auf dem Prinzip einander einschreibender Kreise und Quadrate. So hat ein das Quadrat umschreibender Kreis (also ein Kreis, dessen Linie die vier Ecken des eingeschriebenen Quadrats berührt) einen Kreisdurchmesser, der identisch ist mit der Länge der Quadratdiagonalen. Ein demselben Quadrat eingeschriebener Kreis hat dabei als Durchmesser die Seitenlänge des Quadrats.

Dieses Schema hat man lange für ein zentrales Entwurfsschema der mittelalterlichen Architektur gehalten; es läßt sich jedoch vorwiegend für die Entwicklung architektonischer Kleinformen nachweisen. Vgl. P. Pause: Gotische Architekturzeichnungen in Deutschland, Phil. Diss., Bonn 1973; K. Hecht: Maß und Zahl in der gotischen Baukunst, Hildesheim 1979; A. Seeliger-Zeiss: Studien zum Steinmetzbuch des Lorenz Lechler von 1516, in: architectura 12,1982, 125-150.

35. Vgl. Zöllner: Vitruvs Proportionsfigur, 77-87.

36. Vgl. Codex Huygens, fols. 1, 5-7, 12-15, 28 und 34 (teilweise reproduziert bei Panofsky: The Codex Huygens, Tf. 1, 5, 7 und 17).

37. Vgl. R. de Maulde la Clavière: Jean Perréal, dit Jean de Paris. Sa vie et son oeuvre, in: Gazette des BeauxArts 14,1895, 265-278, und 15,1896, 58-70, 240-252 und 367-381; L. Dorez: Léonard de Vinci et Jean Perréal, in: Léonard de Vinci 1519-1919. Aux éditions de la Nouvelle Revue d'Italie, Rom 1919, 67-86; P. Durrie: Les Relations de Léonard de Vinci avec le peintre francais Jean Perrèal, in: Etudes italiennes 1,1919, 152-167; Raccolta Vinciana 11,1922, 62-63. E. Verga: Bibliografia Vinciana, 2 Bde., Bologna 1931, Bd. 2, Nr. 2441 und 2466. Thieme/Becker: Allgemeines Lexikon der bildenden Künstler, Bd. 26, Leipzig 1932, 433-435 (F. Winkler); Ch. Sterling: Une peinture certaine de Perréal enfin retrouvée, in: L'Oeil, Nr. 103-104, July-August 1963, 2-15, 64f; G. Kauffmann: Die Kunst des 16. Jahrhunderts (Propyläen Kunstgeschichte Band 8), Berlin 1970, 225f, 234f und passim.

38. Abbbildung bei De Maulde la Clavière: Jean Perréal, 2 (Gazette des Beaux-Arts 15,1896, 67), und bei Dorez: Léonard de Vinci et Jean Perréal, 74.

39. Richter: Literary Works, §1379.

40. Vgl. A. Vernet: Jean Perréal, poète et alchimiste, in: Bibliothèque d'humanisme et renaissance 3,1943, 214-252, 233-234.

41. Agrippa von Nettesheim: Opera, 2 Bde., Lyon o.J. [ca. 1630], Bd. 2, 697-698, Ep. 1.17; 811-812, Ep. 3.54; 901, Ep. 5.8; vgl. auch 900, Ep. 5.7.

42. ebd., 905. Ep. 5.14, vom 24.9.1527.

43. Luca Pacioli: De divina proportione, Venedig 1509, zit. nach: Scritti rinascimentali di architettura. A cura di A. Bruschi, C. Maltese etc., Mailand 1978, 23-144, 57. 
44. "Parimenti di poco frutto fù, e di poca sostanza, altra regola che lasciò disegnata con scritti alla rouerscia altro pur valent'huomo di professione, ma troppo sofistico anch'egli, in lasciare precetti pur mathematici à muouere e torcere la figura con linee perpendicolari, con squadra, e compassi." Federigo Zuccari: Idea de' pittori, scvltori, et architetti, Turin 1607 (Nachdruck Florenz 1961), 31; vgl. hierzu zuletzt A. Vezzosi (Hrsg.): Leonardo e il leonardismo a Napoli e Roma, Florenz 1983, 232.

45. Leonardo da Vinci: Codices Madrid, Ed. L. Reti, 5 Bde., Frankfurt 1974 (Rez. J. S. Ackerman, in: Journal of the Society of Architectural Historians 36,1977, 46-50); vgl. auch L. Reti: The Two Unpublished Manuscripts of Leonardo da Vinci in the Biblioteca Nacional, in: The Burlington Magazine 110,1968, 10-22; L. Heydenreich: Bemerkungen zu den zwei wiedergefundenen Manuskripten Leonardo da Vincis in Madrid, in: Kunstchronik 21,1968, 85-100.

46. Diese Phase ist durch folgende Zeichnungen dokumentiert (etwa in ihrer chronologischen Reihenfolge): Windsor 19140r, 12607r, 12601r; Accademia, Venedig, Nr. 236v und 236r; Windsor 19129r, 19135r, 19124r, 19133r, 19136-139v, 19136-139r, 19130v, 19130r, 19131r, 19132r, 19133v, 19131v; Paris, Ms. A, fol. 63r, Ms. Ash. 2038, fol. 23v, Ms. Ash. 2038, fols. 29r und 32r; Windsor 12614r.

47. Vgl. Zöllner: Agrippa, Leonardo and the Codex Huygens.

48. Die Verbindungen ergeben sich sowohl aus dem Vitruvischen Proportionssystem als auch aus anderen Details, etwa aus den Meßpunkten der Vitruvstudie, die in den Bewegungsdarstellungen oft zu Bewegungszentren werden, oder aus detaillierteren Proportionen (vgl. Zöllner: Leonardo, Agrippa and the Codex Huygens). Überhaupt macht erst Leonardos Modifikation der Proportionsfigur Vitruvs diese Figur zur Grundlage von Bewegungsstudien; vgl. hierzu z.B. die Entwicklung der Bewegung von der Vitruvzeichnung in Venedig (Abb. 1) zu fol. 1 (Abb. bei Panofsky: Codex Huygens, Abb. 1) und fol. 12 (Abb. 7) des Codex Huygens.

49. Leonardo da Vinci: Das Buch von der Malerei, Ed. Ludwig, 3 Bde., Wien 1882 (Nachdruck Osnabrück 1972), Nr. 278-279, 309-310, 313 und 345, 348, 392, 394 (im folgenden zit. als Leonardo: Trattato, Ed. Ludwig). Alle datierbar zwischen 1500 und 1510; vgl. die Konkordanz bei Pedretti: Libro A, 188-201.

50. Vgl. Codex Urbinas, fol. 59r, (Ed. Ludwig, Nr. 175; Leonardo da Vinci: Treatise on Painting, Ed. McMahon, 2 Bde., Princeton 1956, Nr. 125; bei Richter: Literary Works, §359) d.i. Ms. A, fol 29r, Del colochar le figure.

51. Leonardo: Trattato, Ed. Ludwig, Nr. 318 (d.i. Codex Urbinas, fol. 113v-114r; ca. 1508-1510, vgl. Pedretti: Libro A, 191; Pedretti: Commentary, Bd. 1, 267.)

52. Leonardo: Trattato, Ed. Ludwig, Nr. 397 (vgl. Pedretti: Libro A, 195, hinsichtlich der Datierung)

53. Vgl. Aristophanes: Nubes, 202-204; Cassiodorus Senator: Varia, 3.52; Isidor von Sevilla: Etymologiarum libri XX, 3.10.1-3;

54. Leonardo: Trattato, Ed. Ludwig, Nr. 33.

55. Vgl. z.B. Codex Atlanticus, fol. 199v-a (ca. 1505-1508); Richter: Literary Works, Bd. 1, Pl. XXXI.

56. Vgl. Panofsky: Codex Huygens, 122-124. 
57. Codex Urbinas, fol. 130r (d.i. Libro A, c. 40; Leonardo: Trattato, Ed. Ludwig Nr. 402, datierbar nach Pedretti: Libro A, 195, auf ca. 1508-1510); Paris, Ms. M, fol. 78v (das gesamte Manuskript datierbar vor 1500). Vgl. auch Codex Urbinas, fol. 246r (d.i. Leonardo: Trattato, Ed. Ludwig, Nr. 829, datiert von Pedretti: Libro A, 217, auf 1508-1510).

58. Diese Phänomen bedürfte einer genauen kompositionellen Analyse. Ich nennen hier nur einige Beispiele aus A. E. Popham: The Drawings of Leonardo da Vinci, London 1949, Nr. 9B, 41, 42, 44, 48, 105, 107, 108, 174-176 und 305.

59. Vgl. C. Pedretti: Leonardo. A Study in Chronology and Style, London 1973, 103 und 110; J. Wasserman: A Re-discovered Cartoon by Leonardo da Vinci, in: The Burlington Magazine, 112,1970, 194-204; V. Budny: The Sequence of Leonardo's Sketches for "The Virgin and Child with Saint Anne and Saint John the Baptist", in: Art Bulletin 65,1983, 34-50. 